\title{
Spontaneous healing of osteitis fibrosa cystica in primary hyperparathyroidism
}

\author{
CJ Gibbs, JGB Millar, J Smith
}

\begin{abstract}
Summary
A 24-year-old man with primary hyperparathyroidism and osteitis fibrosa cystica developed acute hypocalcaemia. Spontaneous healing of his bone disease was confirmed radiographically and by correction of the serum alkaline phosphatase. Hypercalcaemia associated with a raised serum parathyroid hormone recurred 90 weeks after the initial presentation. During the fourth neck exploration a parathyroid adenoma was removed, resulting in resolution of his condition. Haemorrhagic infarction of an adenoma was the most likely cause of the acute hypocalcaemic episode.
\end{abstract}

Keywords: primary hyperparathyroidism, osteitis fibrosa cystica, hypercalcaemia

A 24-year-old man presented with a painless swelling of the right mandible, lethargy, anorexia, thirst and lower backache. Examination was normal apart from the mandibular swelling. Radiographs showed a large translucency in the right mandibular body (figure 1A). Subperiosteal erosion and distal phalangeal tuft resorption were present in the hands (figure 2A). Intravenous urography showed localised medullary sponge kidney of the upper pole of the left kidney with nephrocalcinosis. Serum biochemistry showed hypercalcaemia and an elevated parathyroid hormone concentration (table).

Ten days after the initial consultation the patient was admitted with paraesthesiae in the

Department of

Endocrine Medicine, Southampton University and St Mary's Hospital, Portsmouth, UK

CJ Gibbs

JGB Millar

\section{Department of} Pathology, St Mary's Hospital, Newport, Isle of Wight, UK J Smith

Correspondence to $\mathrm{Dr} \mathrm{CJ}$ Gibbs, Department of General and Endocrine General and Endocrin Medicine, Greenwich Hill, Greenwich, London SE10 9HE, UK

Accepted 24 January 1996 hands, feet and face, and dyspnoea from a feeling of pressure on his chest. Serum biochemistry showed hypocalcaemia (table).

$\mathrm{He}$ was treated with oral calcium supplements $1200 \mathrm{mg}$ daily (without vitamin D) and discharged home. A biopsy of the mandibular swelling one month later showed a 'brown tumour' due to hyperparathyroid bone disease. There was fibrous replacement of the marrow space (osteitis fibrosa cystica). Osteoclasts were numerous and osteoblastic new bone formation was increased at the margins of the lesion.

His backache gradually improved; by 30 weeks the serum calcium returned to normal and the serum alkaline phosphatase fell to just above the upper reference limit (figure 3 ). $\mathrm{He}$ remained well on no treatment until he presented again with the original symptoms 90 weeks after the first consultation. Serum biochemistry showed hypercalcaemia, hypophosphataemia, elevated parathyroid hormone, but normal alkaline phosphatase (table). Radiographs showed improvement in the mandibular translucency and resolution of the phalangeal tuft resorption and subperiosteal erosion (figures 1B, 2B). Thallium scan of the neck showed no evidence of parathyroid activity and neck exploration failed to reveal any parathyroid tissue. Venous sampling showed no step-up in parathyroid hormone concentration in the neck or chest. Selective angiography suggested a parathyroid adenoma behind the right clavicle but two further explorations revealed only one normal parathyroid gland. Computed tomography (CT) of the neck showed a low attenuation, nonenhancing mass in the right lower pole of the thyroid gland. Ultrasonography confirmed a hypo-echoic mass $1.5 \times 0.5 \mathrm{~cm}$ in the right lobe of the thyroid.

Removal of the right lobe of the thyroid during the fourth neck exploration, five years after the first presentation, was successful and histology confirmed a parathyroid adenoma $1.4 \times 1.0 \times 0.8 \mathrm{~cm}$ within the excised thyroid tissue. The patient became hypocalcaemic and required maintenance oral calcitriol treatment for hypoparathyroidism, but he remained well two years later on calcitriol $6 \mu \mathrm{g} /$ day with a serum calcium in the normal range.

\section{Discussion}

This is the first well-documented report of a spontaneous cure of osteitis fibrosa cystica in $ᄋ$ proven primary hyperparathyroidism. The acute hypocalcaemia that heralded the event was most probably due to haemorrhagic infarction of a parathyroid adenoma, which could be termed parathyroid apoplexy. Similar crises are well known in other endocrine adenomata, for example, pituitary apoplexy. We could not justify urgent surgical explora- 0 tion to prove the presence of a necrotic parathyroid adenoma and at that date urgent CT, ultrasound or magnetic resonance imaging of the neck to demonstrate a haemorrhagic mass were not available at the unit where he presented. When hyperparathyroidism recurred and a parathyroid adenoma was excised, no infarcted parathyroid tissue was 8 found, but this is not surprising in view of the interval of five years. A previous pathological $\overline{0}$ study has shown that severe acute hypocalcae- ? mia in a patient with a suspected parathyroid 
Figure 1 Radiographs of the mandible (A) on presentation, (B) 10 months laters and (C) five years later, showing spontaneous resolution of osteitis fibrosa cystica. Note the translucency due to a brown tumour eroding the cusps of the adjacent molar (A). Healing is apparent in (B) with new bone formation within the defect. In (C) the lesion has reduced in size and scleroris is apparent in the posterior half of the brown tumour. A dense sclerotic rim is extending into the lesion from the margins

Figure 2 Radiographs of the hand showing distal phalangeal tuft resorption and subperiosteal resorption along the radial borders of the phalanges (A) and spontaneous resolution after two years (B)
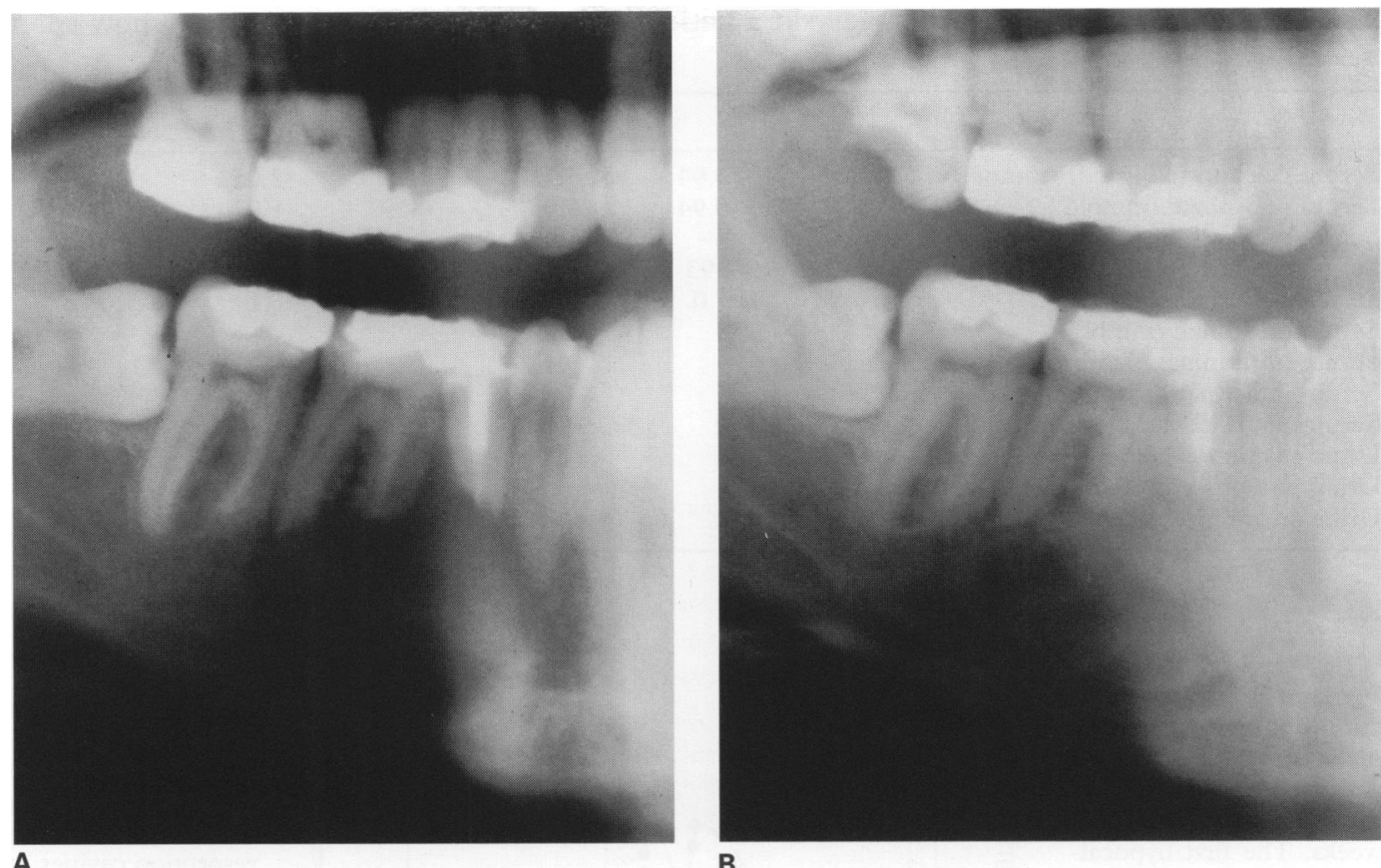

B
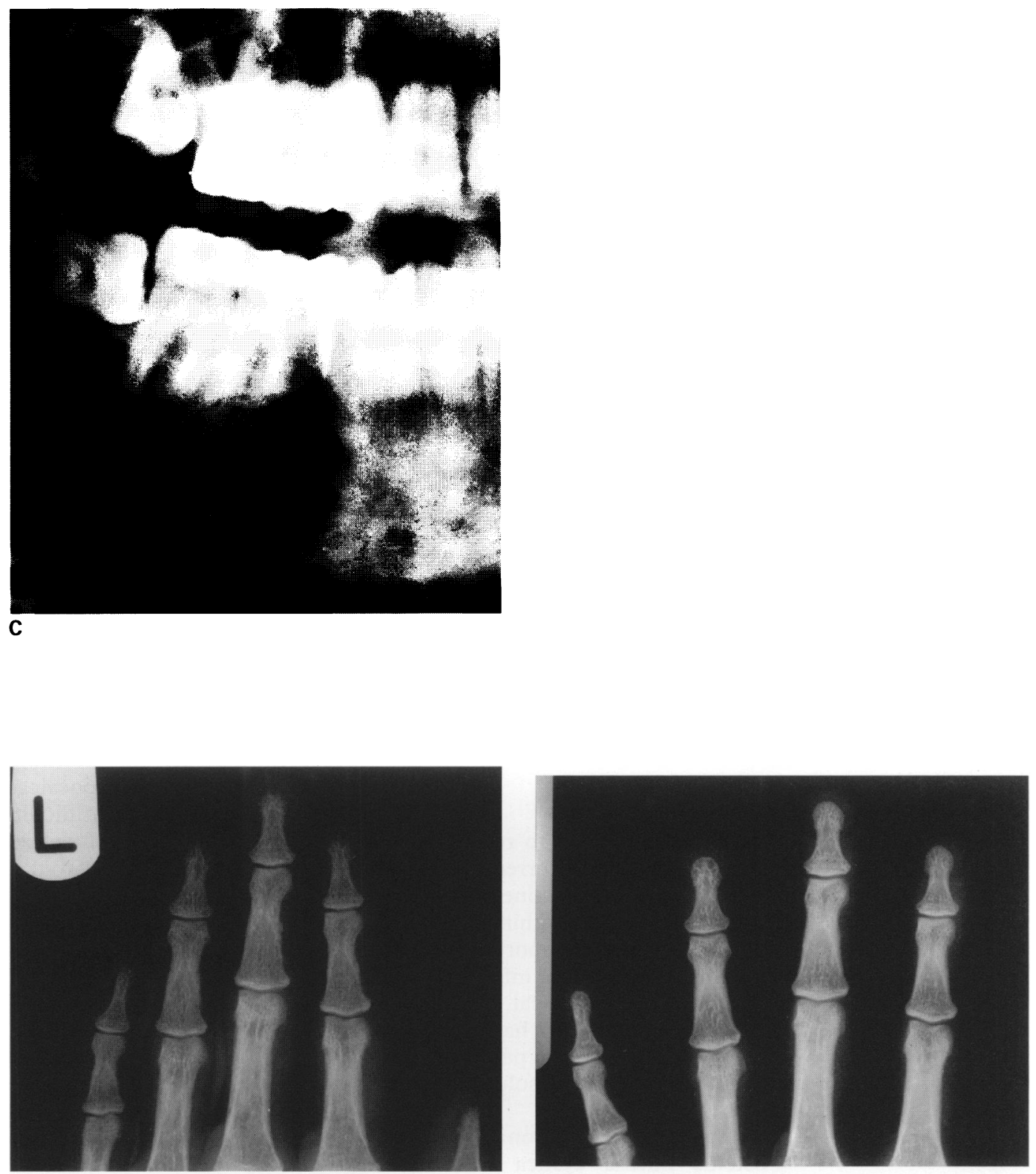

B

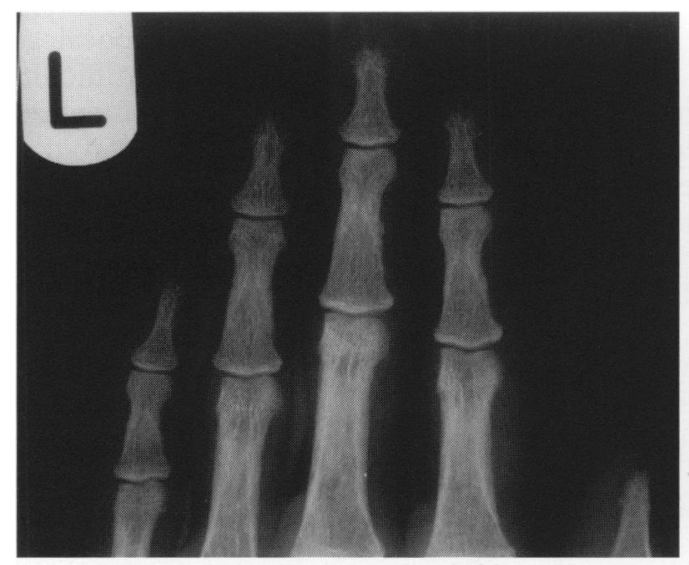


Table Serum and urine biochemistry in a patient with a parathyroid adenoma to show spontaneous changes over 90 weeks

\begin{tabular}{|c|c|c|c|c|c|c|c|}
\hline Weeks: & 1 & 2 & 3 & 12 & 30 & 90 & Normal range \\
\hline Serum calcium (adjusted) $(\mathrm{mmol} / \mathrm{l})$ & 3.94 & 3.71 & 1.78 & 2.05 & 2.23 & 3.25 & $2.20-2.60$ \\
\hline Serum phosphate (mmol/l) & 0.94 & 0.85 & 0.59 & 1.07 & 1.37 & 0.56 & $0.80-1.5$ \\
\hline Serum magnesium (mmol/l) & - & 0.94 & 0.74 & - & 0.67 & 0.84 & $0.7-1.2$ \\
\hline Serum alkaline phosphatase (IU/l) & 993 & 1041 & 925 & 633 & 298 & 269 & $80-280$ \\
\hline Serum urea $(\mathrm{mmol} / \mathrm{l})$ & 7.0 & 7.3 & 8.6 & 6.1 & 7.0 & 5.1 & $2.0-6.5$ \\
\hline Serum creatinine $(\mathrm{mmol} / \mathrm{l})$ & - & 121 & - & - & - & 120 & $60-130$ \\
\hline Serum N-terminal parathyroid hormone $(\mathrm{ng} / \mathrm{ml})$ & - & 3.4 & 0.7 & - & - & - & $<0.6$ \\
\hline Serum intact parathyroid hormone (pmol/l) & - & - & - & - & - & 19.3 & $0.8-8.5$ \\
\hline Serum $250 \mathrm{H}$-cholecalciferol $(\mathrm{ng} / \mathrm{ml})$ & - & - & 8.0 & - & 13.8 & - & $8-50$ \\
\hline Urine calcium excretion (mmol/24 h) & - & - & 0.2 & - & - & 4.6 & $1.5-7.5$ \\
\hline Urine phosphate excretion (mmol/24 h) & - & - & 3.4 & - & - & 23 & $16-32$ \\
\hline Urine creatinine excretion (mmol/24 h) & - & - & 4.6 & - & - & 11.4 & $9-17$ \\
\hline
\end{tabular}

Figure 3 Serum calcium and serum alkaline phosphatase in a patient with a parathyroid adeno$\mathrm{ma}$ followed for 250 weeks. The first hypocalcaemic episode was spontaneous, the second was after parathyroidectomy
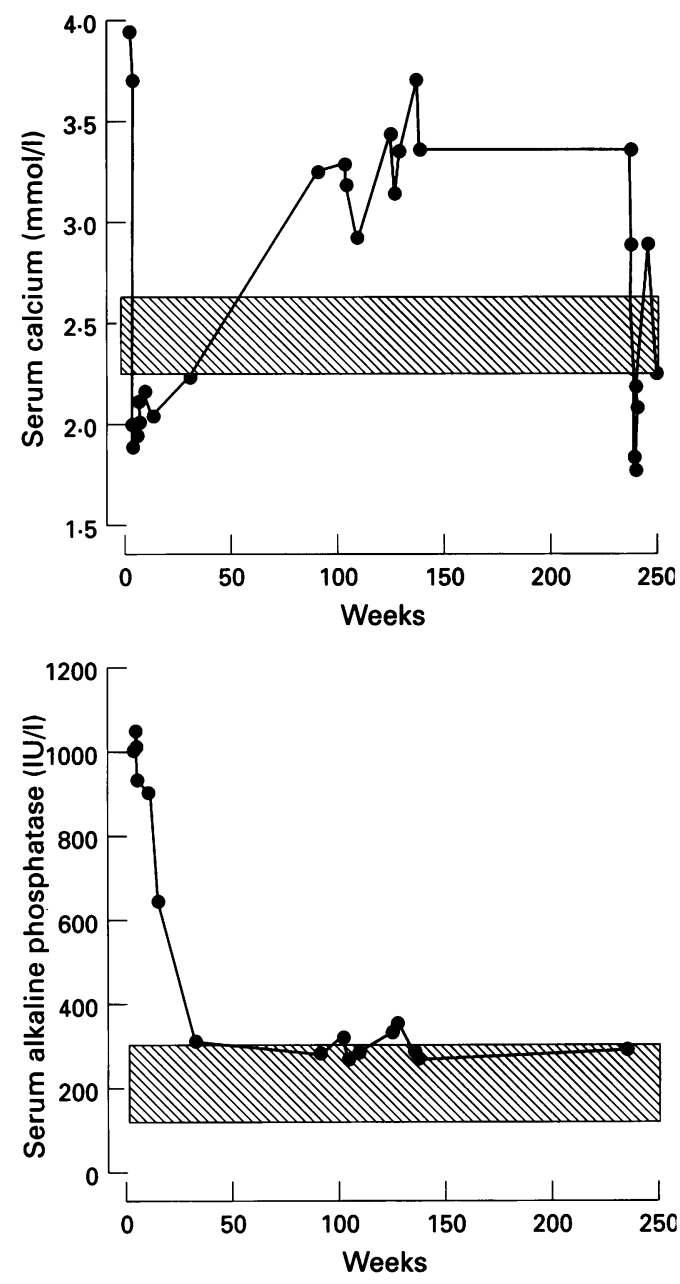

tumour was related to recent infarction of an adenoma. ${ }^{1}$ The recurrence of a parathyroid adenoma can be explained by regrowth of nests of viable chief cells within a necrosed adenoma, as these have been reported in a patient whose parathyroid adenoma infarcted after calcitonin treatment. ${ }^{2}$ Histological proof of necrosis in a parathyroid adenoma has also been described in a patient who had a parathyroidectomy soon after spontaneous resolution of hypercalcaemia. $^{3}$

The lack of synchronicity of the activity of osteitis fibrosa cystica with the degree of hypercalcaemia is interesting, but consistent with the Frost model of bone remodelling. ${ }^{4}$

\begin{tabular}{|l|}
\hline Summary/learning points \\
\hline - after parathyroidectomy hungry bone disease \\
may occur, causing prolonged hypocalcaemia \\
while the skeleton takes up mineral to fill \\
resorption cavities \\
- brown tumours heal by dense sclerosis over 12 \\
months
\end{tabular}

The heightened activity of the osteoclasts up to the time of involution of the adenoma would lead to numerous resorption cavities. The time taken for osteoblasts to fill these cavities with new bone is three to four months in normal $\vec{\theta}$ bone, but may be longer in osteitis fibrosa $\mathscr{\%}$ cystica despite the high bone turnover. The bone formation marker, alkaline phosphatase, took over 30 weeks to return to normal in our case. The mandibular defect healed as would be expected for a brown tumour which typically undergoes dense sclerosis within $12 \overrightarrow{\vec{a}}$ months, ${ }^{5}$ in contrast to bone cysts which do not remineralise. Complete sclerosis did not occur, probably because of recurrent hyperparathyroidism after 30 weeks.

After parathyroidectomy the skeleton's avid requirement for calcium and phosphate to mineralise new bone (hungry bone disease) may last for months. ${ }^{6}$ The prolonged hypocalcaemia in our patient was probably due to this, compounded by suppression of the remaining parathyroid glands by previous chronic hyper- $\frac{D}{O}$ calcaemia, ${ }^{7}$ as demonstrated by an inadequate parathyroid hormone response $(0.7 \mathrm{ng} / \mathrm{ml})$ N during the hypocalcaemic crisis.

There was difficulty in localising the adenoma because of its size and position. Small and ectopic parathyroid glands are poorly visualised by thallium subtraction imaging because of the low energy of the emitted photon. ${ }^{8}$

Finally, hypocalcaemia in a patient with 0 known primary hyperparathyroidism should lead one to consider acute pancreatitis and vitamin $\mathrm{D}$ deficiency, which are known com- $\mathbb{D}$ plications of primary hyperparathyroidism and $\sigma_{2}$ more common causes of hypocalcaemia than necrosis of an adenoma. ${ }^{9}$

We acknowledge the assistance given by Professor JHL O'Riordan, Mr EJG Milroy and Dr HWP Rooke. 
1 Norris EH. Primary hyperparathyroidism. Arch Pathol 1946; 42: $261-73$

2 Minisola S, Romagnoli E, Scarnecchia L, et al. Parathyroid storm: immediate recognition and pathophysiological considerations. Bone 1993; 14: 703-6.

3 Howard JE, Follis RH, Yendt ER, Connor TB. Hyperparathyroidism. Case report illustrating spontaneous remission due to necrosis of adenoma. F Clin Endocrinol Metab 1953; 13: $997-1008$.

4 Frost HM. Bone remodelling and its relationship to metabolic bone disease. Springfield IL: Charles C Thomas, 1973.

5 Aurbach GD, Marx SJ, Spiegal AM. Parathyroid hormone, calcitonin and calciferols. In: Wilson JD, Foster DW, eds, Williams textbook of endocrinology. Philadelphia: WB Saunders, 1985.
6 Norton JA, Aurbach GD, Marx SJ, Doppman JL. Surgical management of hyperparathyroidism. In: De Groot LJ, ed.

End.

7 Brown EM, Wilson RE, Thatcher JG, Marynick SP. Abnormal calcium regulated PTH release in normal parathyroid tissue from patients with adenoma. $\mathrm{Am} \mathcal{F} \mathrm{Med}$ 1981; 71: 565-74.

8. Peck WW, Higgins CB, Fisher MR, et al. Hyperparathyroidism: comparison of MR imaging with radionuclide scanning. Radiology 1987; 163: 415-20.

9 Lumb GA, Stanbury SW. Parathyroid function in human vitamin $D$ deficiency and vitamin $D$ deficiency in primary hyperparathyroidism. Am $f$ Med 1974; 56: 8335.

\title{
Cefuroxime-induced thrombocytopenia?
}

\author{
Peter Aitken, SMN Zaidi
}

\section{Summary \\ We present the case of a 77-year-old man who became thrombocytopenic whilst treated parenterally with cefuroxime in the absence of proven infection and re- covered when the cefuroxime was discon- tinued.}

Keywords: cefuroxime, thrombocytopenia

Since 1966 there have been a number of case reports suggesting a link between cephalosporin therapy and blood dyscrasias, especially thrombocytopenia. ${ }^{1}$ In each case the causal link remains unproven because of the presence of confounding variables and the ethical position that precludes re-exposure of the sensitive individual to confirm the cause and effect. An immunological mechanism has been described in cefotetan-induced thrombocytopenia where potent IgG-cefotetan-dependent antiplatelet antibodies were detected in the patient's serum. ${ }^{2}$ An animal model in the dog has also been described. ${ }^{3}$

\section{Case report}

A 77-year-old man known to suffer from mild Alzheimer's disease and recurrent depressive disorder was admitted with a 10-day history of increasing anxiety and agitation and worsening depression. Examination revealed marked psychomotor agitation, perseverations and verbal stereotypies. His cognitive functioning had deteriorated. He appeared physically ill with a fluctuating conscious level, tachycardia, tachypnoea and profuse sweating. He remained apyrexial.

We diagnosed acute confusional state (delirium ICD 10) superimposed on mild dementia precipitating a relapse of his depressive disorder. We started haloperidol $5 \mathrm{mg}$ bid on admission. Investigations included full blood count, erythrocyte sedimentation rate, midstream urine and urinary electrolytes, liver function tests, calcium, glucose, thyroid function tests, VDRLTPA, three sets of blood cultures, malarial parasites, a chest $\mathrm{X}$-ray and an electrocardiogram.

The significant preliminary result was growth of a Staphylococcus species (unspecified) in one blood culture bottle. We commenced cefuroxime $750 \mathrm{mg}$ tid parenterally. We repeated the full blood count on days one and three of cefuroxime therapy and noted the platelet count to have fallen to $92 \times 10^{9} / 1$. We considered this to be due to presumed infection or a side-effect of medication. On day five of cefuroxime therapy we received the final microbiological report on the blood culture identifying the organism as Staphylococcus epidermidis, a contaminant skin commensal. We stopped cefuroxime and repeated the full blood count days later, finding that the platelet count had risen to $164 \times 10^{9} / 1$. Nine days later it was $325 \times 10^{9} / 1$. The diagnosis was now a severe depressive episode. A course of electroconvulsive therapy was started on the day after

\section{Side-effects of cefuroxime}

- hypersensitivity reactions

- overgrowth of susceptible organisms

- gastrointestinal disturbance

- pseudomembranous colitis

- haematological parameters altered, including decreased haemoglobin concentration, leucopenia, neutropenia

- positive Coombs test

- transient rise in liver function tests

- pain at injection site

- very rare reports of thrombocytopenia 